\title{
LA-UR-21-29892
}

Approved for public release; distribution is unlimited.

Title: Building an Effective Nuclear Safety Culture

Author(s): $\quad$ Schreiber, Stephen Bruce

Intended for: $\quad$ NET run by ORI-1

Issued: 
Disclaimer:

Los Alamos National Laboratory, an affirmative action/equal opportunity employer, is operated by Triad National Security, LLC for the National Nuclear Security Administration of U.S. Department of Energy under contract 89233218CNA000001. By approving this article, the publisher recognizes that the U.S. Government retains nonexclusive, royalty-free license to publish or reproduce the published form of this contribution, or to allow others to do so, for U.S. Government purposes. Los Alamos National Laboratory requests that the publisher identify this article as work performed under the auspices of the U.S. Department of Energy. Los Alamos National Laboratory strongly supports academic freedom and a researcher's right to publish; as an institution, however, the Laboratory does not endorse the viewpoint of a publication or guarantee its technical correctness. 


\section{Building an Effective Nuclear Safety Culture}

Steve Schreiber

Technical Deputy Director of Actinide Operations

October 21, 2021 


\section{What is a Culture?}

"A culture is a way of life of a group of people - the behaviors, beliefs, values, and symbols that they accept, generally without thinking about them, and that are passed along by communication and imitation from one generation to the next."

- It is not a set of procedures or policies

- It is not the management flavor of the day

- It is not something that can be changed quickly with some "training"

- It is how we collectively behave (even when no one is watching) because of our core values

-We have different cultures between Technical Areas, facilities, divisions, work groups, etc. 


\section{Why Do We Need a "Nuclear Safety" Culture?}

- You work in a very unique and hazardous environment

- Significant consequences could result from not following procedures and policies (radiological uptake, equipment/facility damage, environmental insult, dose to the public, nuclear criticality)

- Public Trust

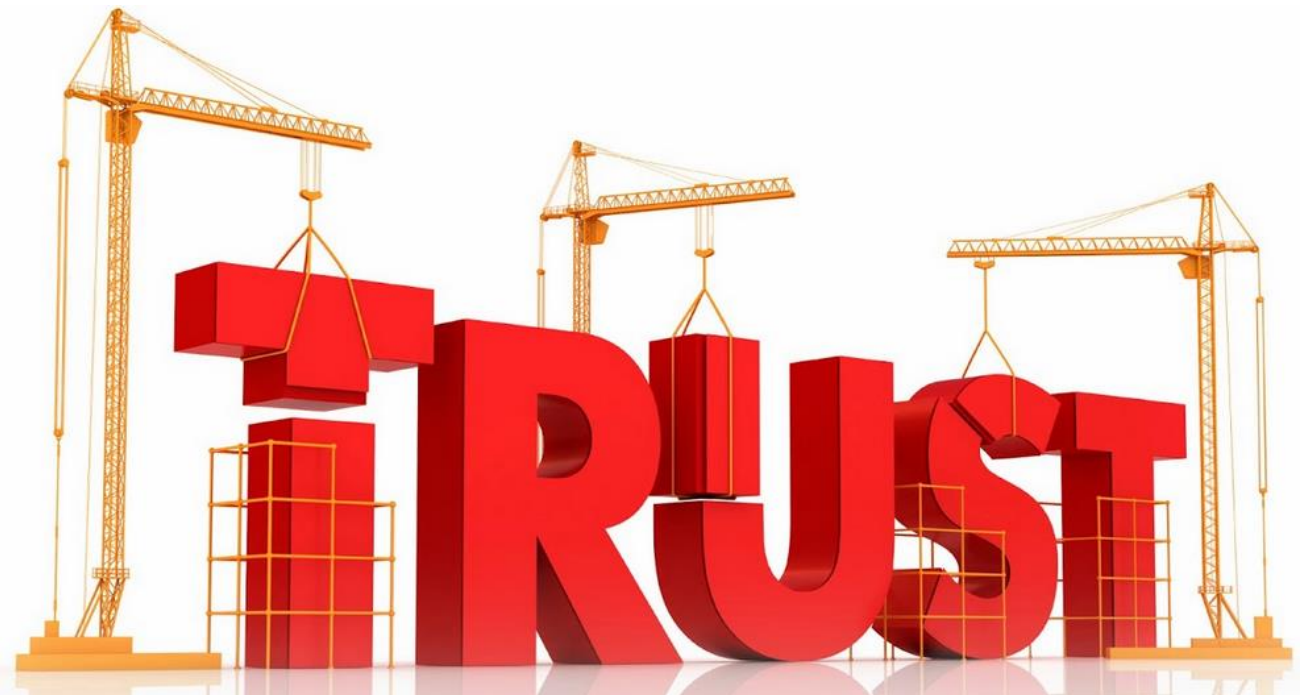




\section{The Battle for Public Opinion is Waged in the Media}

\section{Why should anyone trust LANL on nuclear safety?}

Report: Triad had serious deficiencies in first year running Los Alamos lab

Beyond Nuclear Fears New Mexico Being Targeted For Permanent High-Level Nuclear Waste Dump
Nuclear Winter May Bring a Decade of Destruction
Q Los Alamos REPORTER

Letter To The Editor: No Environmental Review For LANL Expansion
Secrecy News

NNSA Moves to Expand Plutonium Pit Production

DOE Report: Los Alamos National Laboratory Mismanaged Deadly

Controlled Substances

Report: National laboratory lost track of nuclear waste (Associated Press)
MY VIEW

'Aura of apartheid' at LANL offers false hope Plutonium found in glovebox during cleanup
DNFSB Resident Inspectors At LANL Report Waste Shipments Briefly Suspended in November

LANL's seismic safety questioned; lab says improvements are being made 


\section{How does this impact $\underline{u s}$ ?}

- People are watching what happens here and are ready to pounce whenever the opportunity arises

- We work for the government - our missions and funding are dependent on support from Congress

- Our elected officials care about public opinion

- Your livelihood depends on the Laboratory's legacy and reputation
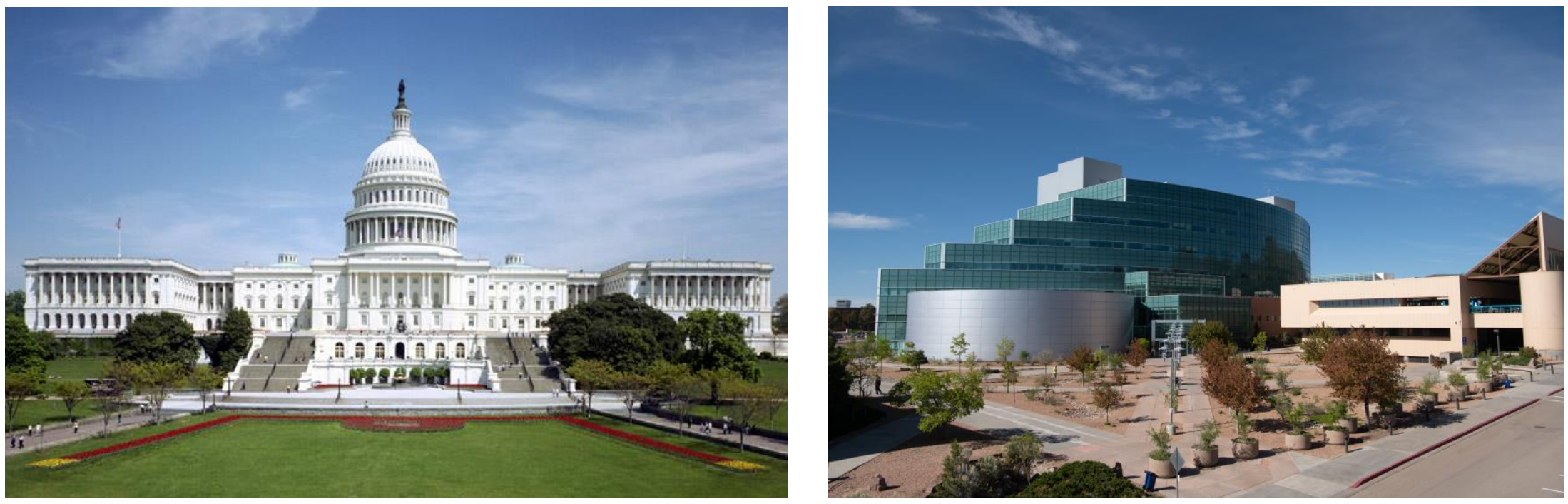


\section{Traits of a Healthy Nuclear Safety Culture}

\section{Established by the Institute of Nuclear Power Operators (INPO)}

"We are united in our pursuit of excellence through a commitment of these values:"

- Trusting Relationships

- Impeccable Integrity

- Influential People

- Unwavering Standards

- Responsible Stewardship

- Innovative Improvement

- INPO 2021
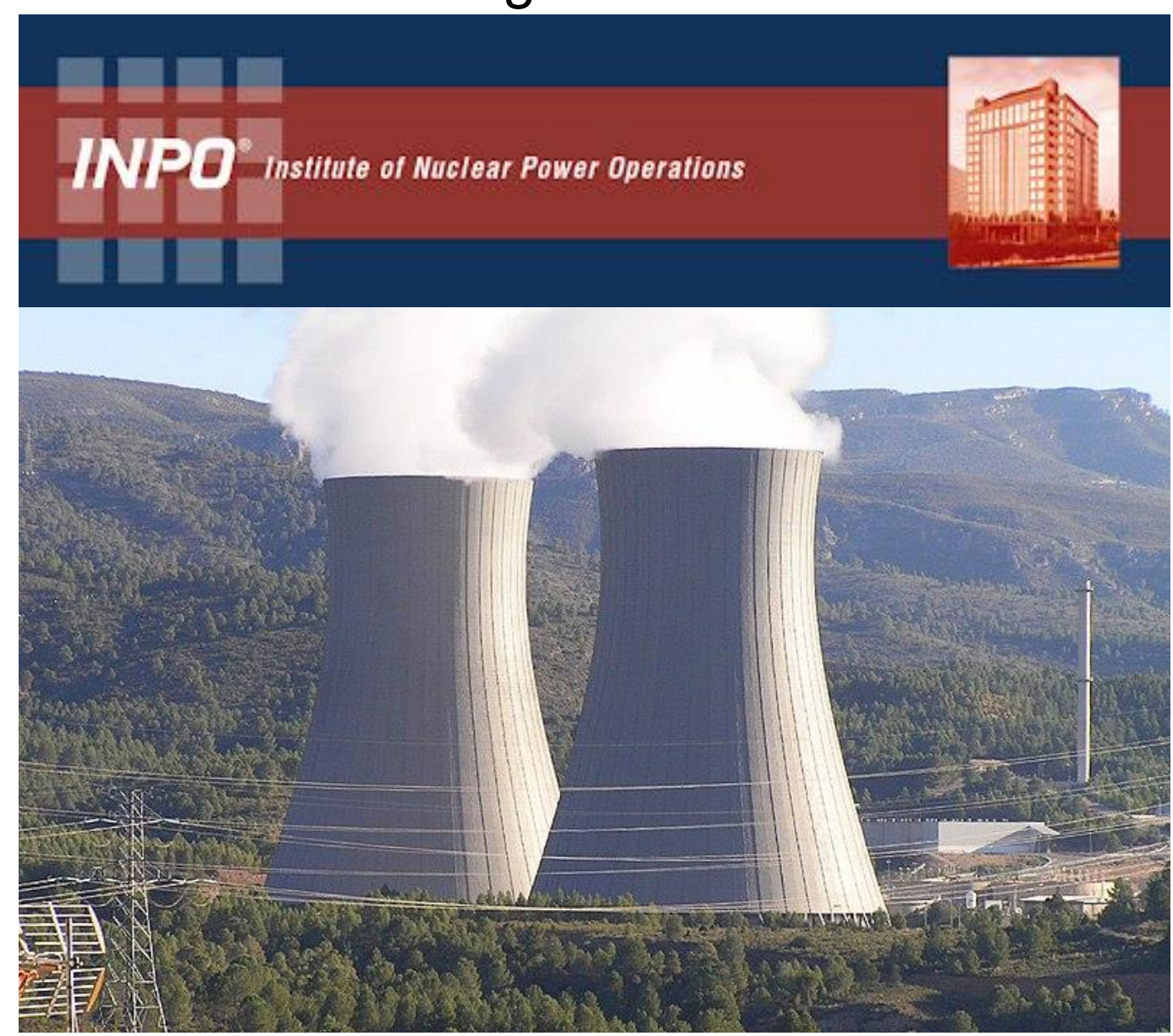


\section{Mission}

To solve national security challenges through simultaneous excellence.

\section{Vision}

To be trusted by our nation, emulated by our peers, and respected by the world.

\section{Culture}

How we do work is as important as what we do.

\section{Simultaneous}

Excellence in... 


\section{LANL Safety Policy}

- "We make safety and security integral to everything we do"

- Safety is not more important than the work we get paid to perform in the national interest, but is a key and requisite part of getting the job done

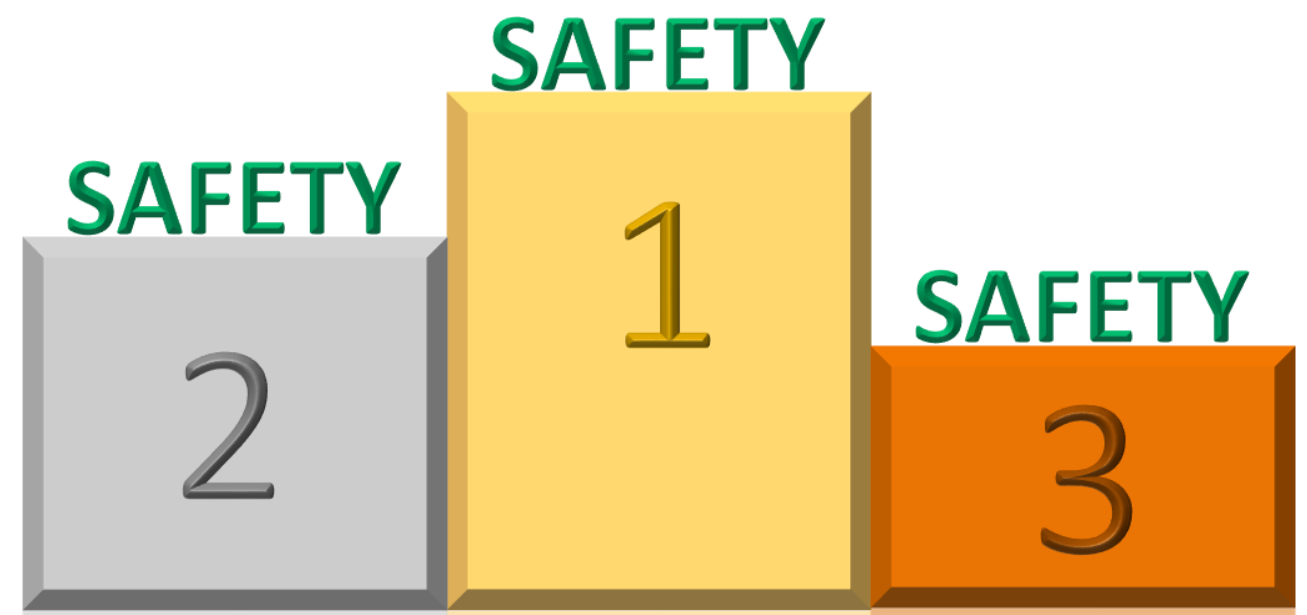




\section{Risk and Safety}

- Safety and risk are two sides of the same coin

- Just to avoid all risk is not necessarily to be safe

- Managing safety and risk is not "black or white" but varying shades of grey

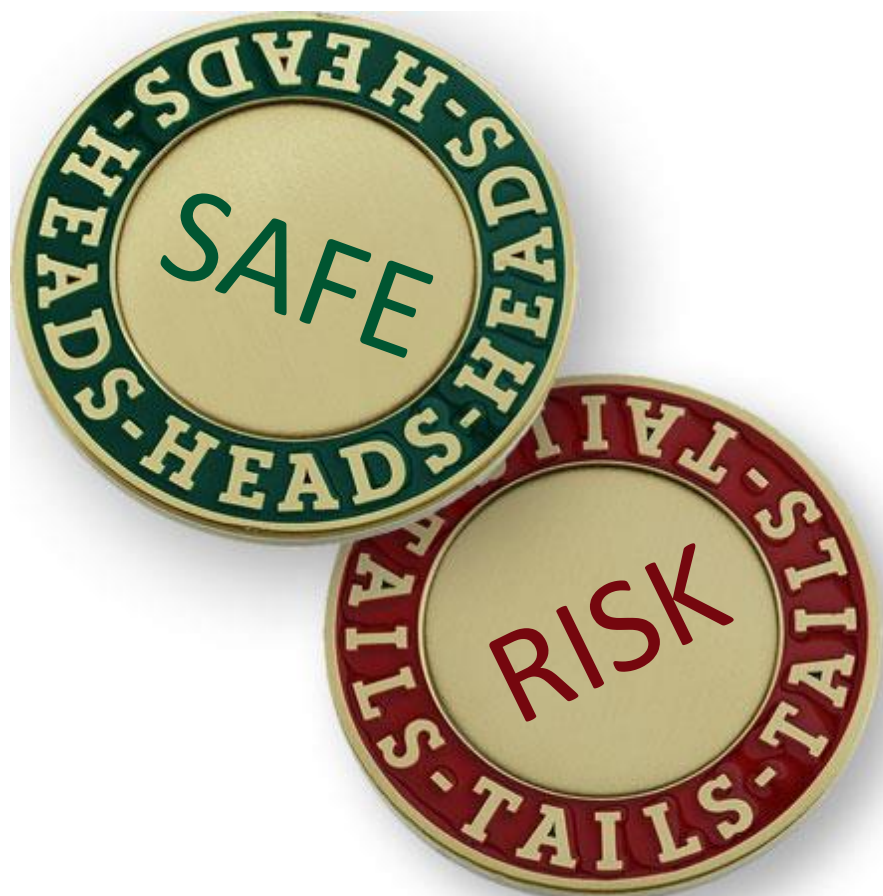




\section{How do we deal with Risk?}

- Risk in general

- Risk aversion in the Nuclear Weapons Complex

- Risk in historical perspective

- Consequences of risk avoidance and the impacts on safety

- Proposed path forward 


\section{Risk (definitions)}

- The chance of death before our allotted time (dictionary)

- Product of the probability and the value of the loss (insurance)

- Product of probability and consequence (to both cost and schedule) per DOE Guide 413.3-7A 


\section{Perception of Risk}

- Western Educated Industrialized Rich Democratic (WEIRD) societies have recently developed the idea that the world owes us all a riskfree life

- When accidents do occur, one should be compensated appropriately which assumes that someone or some "deep pocketed" organization is at fault

- Avoiding Risk then becomes the dominate factor in any decision 


\section{Management of Risk}

- Regulation is the recent preferred weapon against technological risk

- In the United States (as of 1992)

Occupational Safety and Health (OSHA) of the Department of Labor; Consumer Product Safety Commission (CPSC); Environmental Protection Agency (EPA) with offices devoted to water, air, wastes, pesticides, radiation, etc.; Food and Drug Administration (FDA) of Department of Health and Human Services; Nuclear Regulatory Commission (NRC); Federal Emergency Management Agency (FEMA); Bureau of Alcohol, Tobacco, and Firearms (ATF) of the Treasury Department; Mine Safety and Health Administration (MSHA) of the Department of Labor; National Highway Transportation Safety Administration (NHTSA) of the Department of Transportation; Federal Aviation Administration (FAA) of the Department of Transportation along with the National Transportation Safety Board (NTSB) an independent agency; Drug Enforcement Administration (DEA) of the Department of Justice; Cost Guard (USCG) of the Department of Transportation; and the Defense Nuclear Facilities Safety Board (DNFSB). 


\section{Anticipation vs. Resilience}

- Finite or limited resources used to address hypothetical risks or "black swan events" are not available for attaining other goals, goals which indirectly may also lower risks and make us safer

- The number and magnitude of hypothetical harms are limitless which makes great expenditures on them dangerous

- In hindsight after a bad event, it is easy to say that it could have and should have been prevented, but it is also easy to forget or ignore how many hazards that were predicted never came to pass

- Example: At LANL the Plutonium Facility seismic upgrades, specifically the addition of a drag strut across the roof of PF-4, part of \$33M that was unavailable for reinvestment in other 40 year-old infrastructure 


\section{LANL - Plutonium Facility Seismic Upgrade 2011}

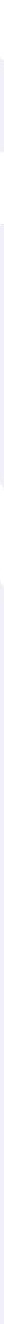

\section{Q LOS Alamos}




\section{Risk in the Modern World}
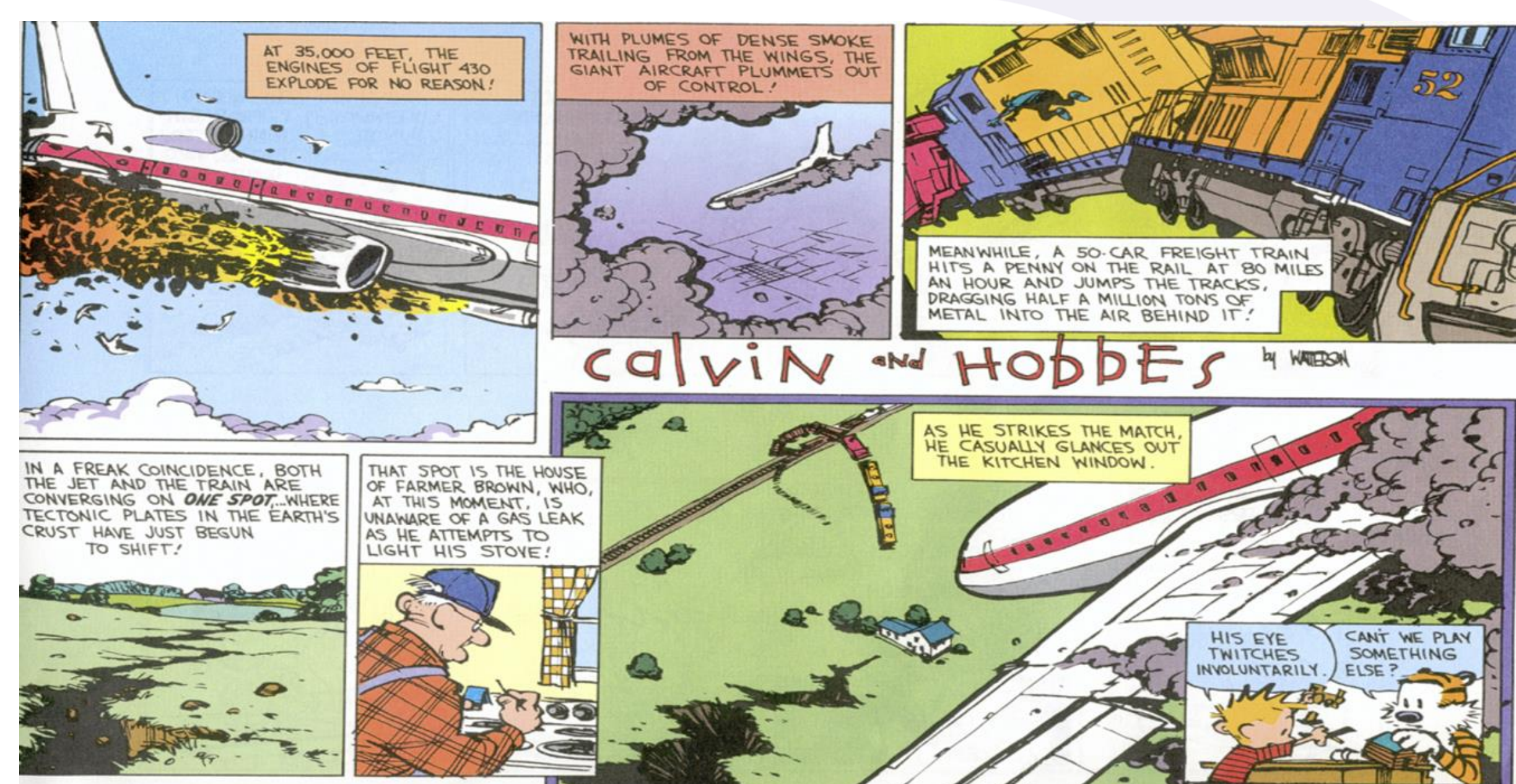

ron-

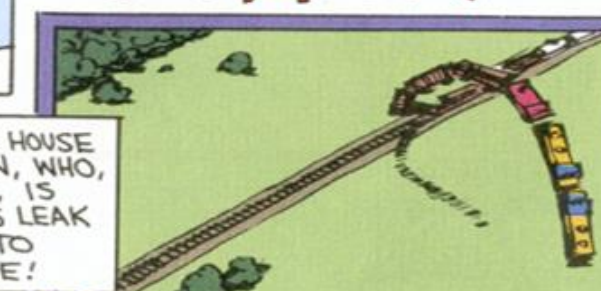

Nd HODDES M W

THAT SPOT IS THE HOUSE OF FARMER BROWN, WHO, UNAWARE OF A GAS LEAK AS HE ATTEMPTS TO
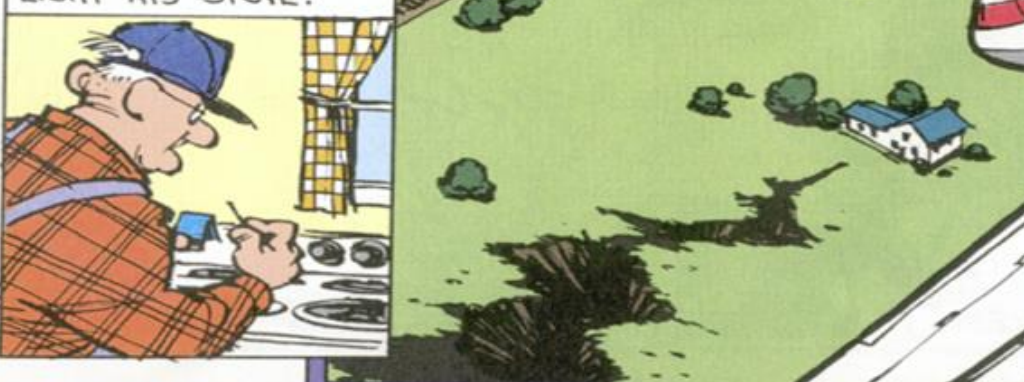

AS ME STRIKES THE MATCH, THE KITCHEN WINDOW.
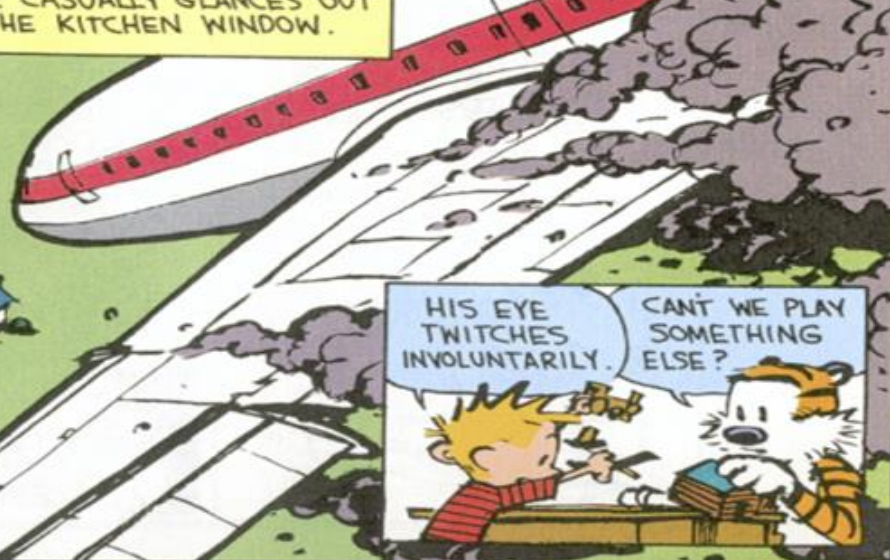

HIS EYE

CANT WE PIAY IN VOLUNTARILY. SOMETHING NMCXS है 12 


\section{Program Requirements vs Regulations}

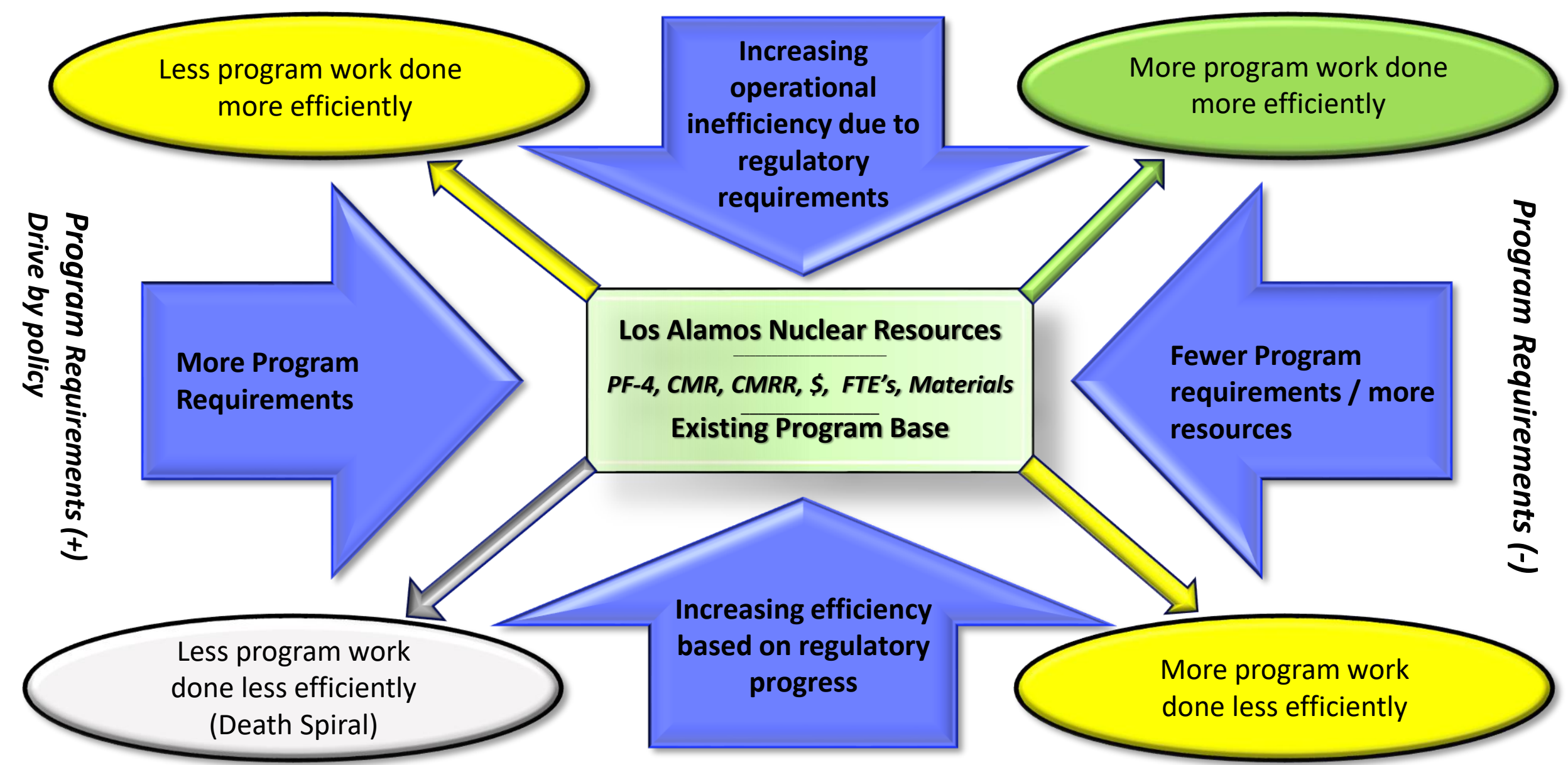

Regulatory Axis (-) 
- Material control and accountability

- Two-person rule

- Periodic SNM Inventory

- Daily Activity Check

- Tamper-indicating devices

- Physical security

- Classified information protection

- Computer security

- Vulnerability analysis

- Human reliability program

- Clearances

- Escorting requirements

- Facility access control

- Material receipt control

- Seismic considerations

- Ventilation operation

- Material-at-Risk (MAR) limits

- Conduct of operations

- Technical surveillance requirements

- Engineering requirements

- Training and qualifications

- Configuration and document control

- Waste acceptance requirements
- Permits (RCRA, NEPA, air, and water)

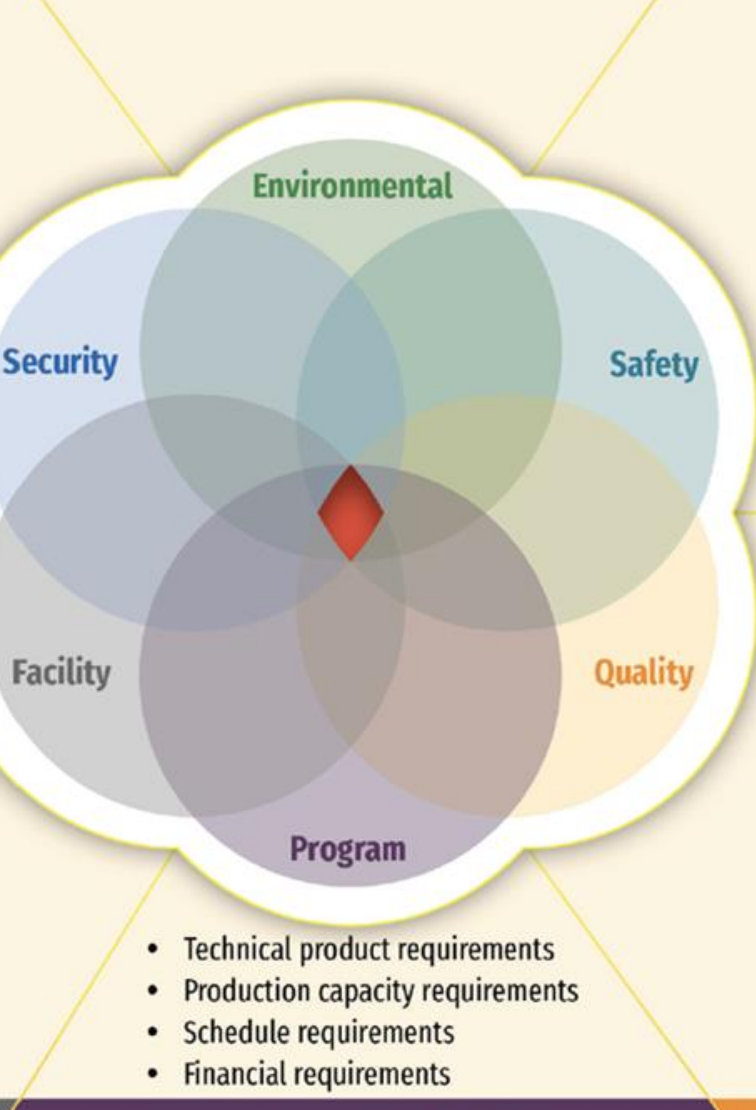

- Financial requirements

- Nuclear criticality

- Radiation protection

- As Low As Reasonably Achievable

- Contamination control

- Dose management

- Industrial safety

- Chemical safety

- Fire detection

- Fire suppression

- Electrical safety

- Laser safety

- Pressure safety

- Facility safety

- Material and test equipment

- Design control

- Process control

- Equipment calibration

- Software quality assurance

- Procurement

- Controlled storage

- Training and certification

- Quality assurance/quality control

Facility Requirement Categories

Program Requirement Categories

Quality Requirement Categories

Recurring Regulatory Issues for Nuclear Facilities

- Number of regulations - cost of compliance - Regulatory independence from resources - Interpretation at multiple levels

Nuclear Safety Culture lies at the intersection of all the requirements for example: a Security Cat I, Hazard Category 2 facility (PF-4) 


\section{WWII Era Safety Message}

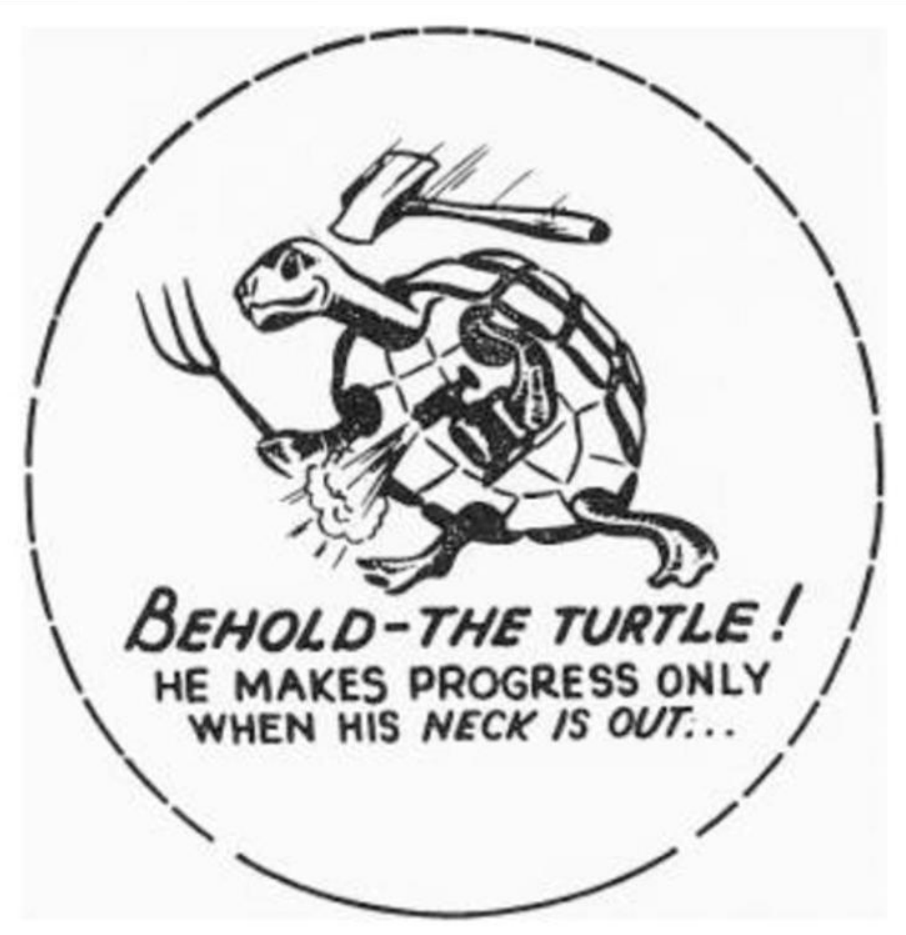

P L A T E 6 A cartoon distributed at Manhattan Project sites during 1944. The purpose of the image, according to General Leslie R. Groves, was "to impress on all concerned the necessity of making decisions promptly and that a 'safety first' policy would insure defeat." Correspondence and Related Papers on the MED, 1942-70, Papers of General Leslie R. Groves, National Archives and Records Administration. 


\section{WWII Era Safety Message}

"Each employee can do more to protect himself and his fellow workers than all the rules in the world, and it is of utmost importance that each of us feel at all times the responsibility of safeguarding himself and others."

- J. Robert Oppenheimer, Los Alamos Laboratory Director, 1945

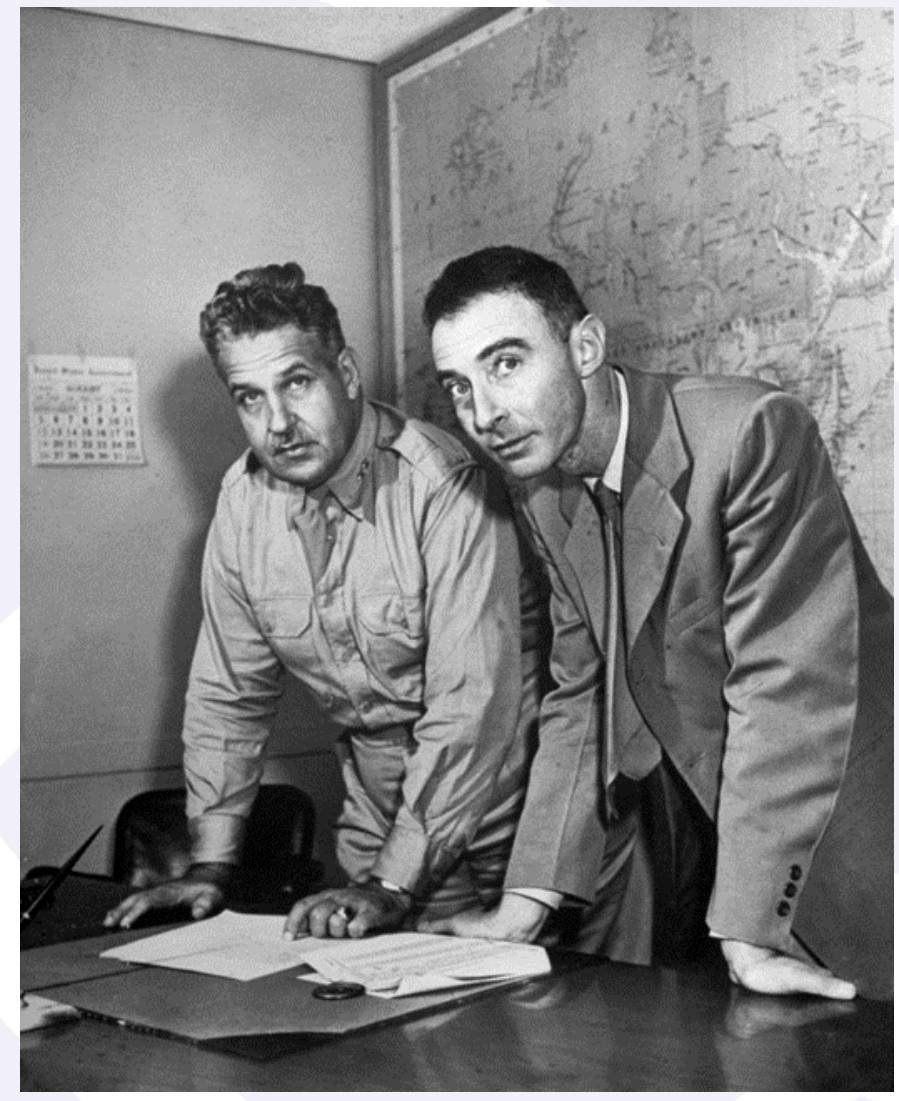

General Leslie R. Groves J. Robert Oppenheimer 


\section{Director Siegfried Hecker Before the HASC on February 16, 2012}

"The stifling oversight is a result of the loss of balance between mission requirements and regulatory/oversight requirements. Congress, apparently in an attempt to enhance the accountability of the Labs and their contractors, has driven the entire system of Laboratory operations toward risk aversion without sufficient consideration for the impact on mission and cost."

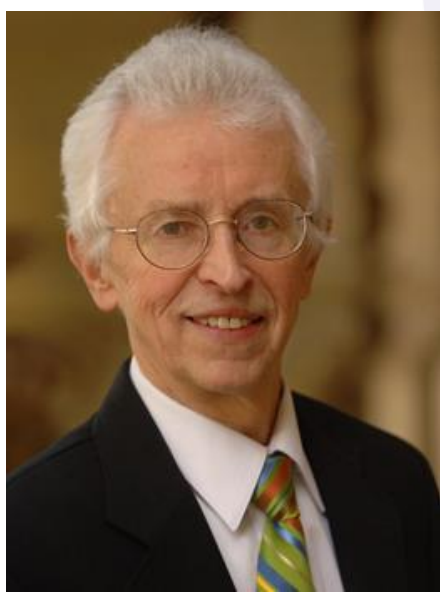

"The primary price the U.S. is paying for risk aversion is not in dollars, but rather in the loss of intellectual capital and the know-how at the Laboratories."

- Director Siegfried Hecker, 2012 


\section{Director Siegfried Hecker Before the HASC on February 16, 2012}

"The Labs are in a state of morale crisis brought on by a suffocating regulatory and operational climate of risk."

"I believe the balance between mission requirements and regulatory requirements has swung so heavily in the direction of the latter that it now seriously endangers the conduct of the nuclear weapons mission in the country's nuclear weapons complex."

"The nature of the nuclear enterprise involves risks - these risks must be managed in a cost effective manner, not avoided by an overly prescriptive and stifling system of multiple layers of oversight."

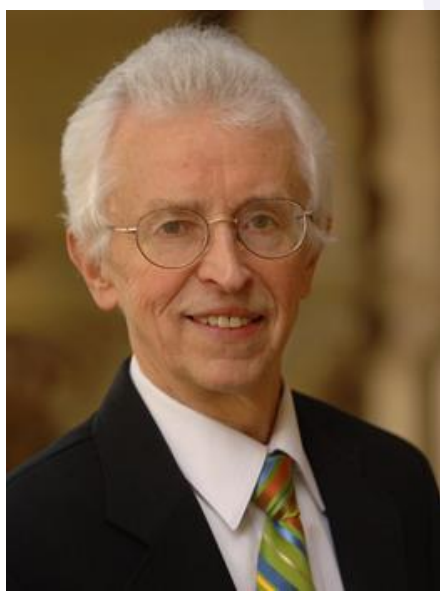

- Director Siegfried Hecker, 2012 


\section{Director Charles McMillan Before the SASC on April 9, 2014}

"We have seen very little relief in the mountain of oversight reviews we must support. Risk aversion among our partners is driving our safety mandates to the point where actually doing work is becoming increasingly difficult. More generally, simply trying to gain permission to build a facility or execute a work scope has become problematic because the many layers of permissions now routinely generate a 'non-concur' that stops the process."

- Director Charles McMillan, 2014

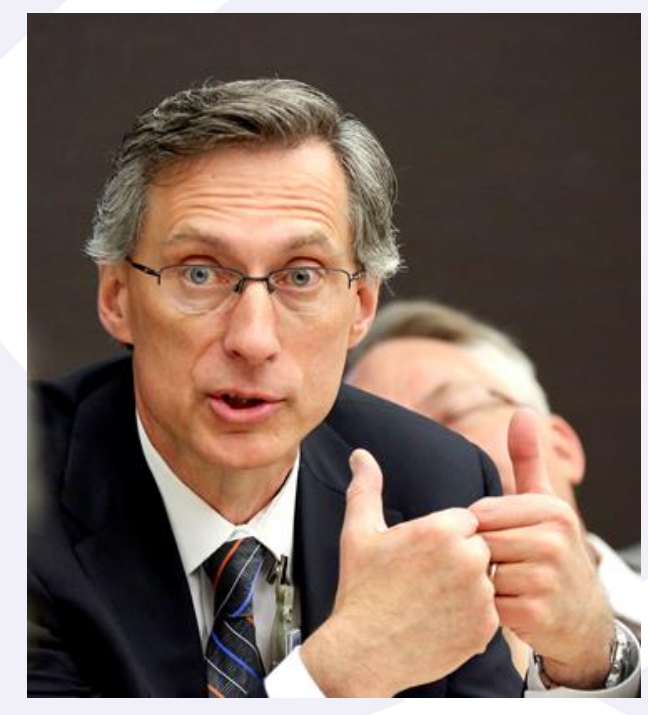




\section{Perils of Risk Avoidance}

- In the field of safety, the law of diminishing returns operates with peculiar ferocity

- If absolute standards are imposed, the attempt to achieve them become intolerably expensive

- Asymptotic curve (probably logarithmic) risk avoidance vs. costs

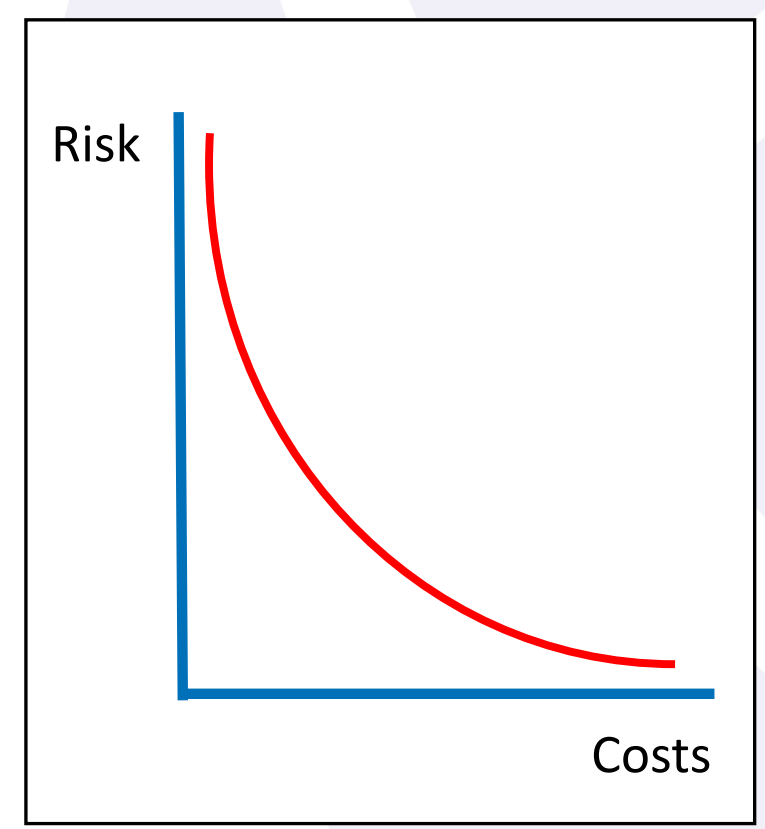




\section{Preparing for the Worst}

- If workers are forced to practice for all possible emergencies or conversely for only the worst conceivable, they will be less well prepared for those few that are more likely

- Worst-case fixation leads to failure in the better-than-worst world

- Finding the worst-case in any realistic scenario is (impossibly) difficult

- Risk without considering probability is just speculative consequence

- Pervasive to assume that if the worst-case is covered, everything else is too 


\section{What is wrong with preparing for the worst?}

- Thought experiment: once an accident situation is identified and the worst case scenario is postulated, then someone else can always invent a worse one

- Design Basis Accidents evolve

- Postulated accidents do not have to be reasonable, only worse

- "Conceivable does not equal credible"
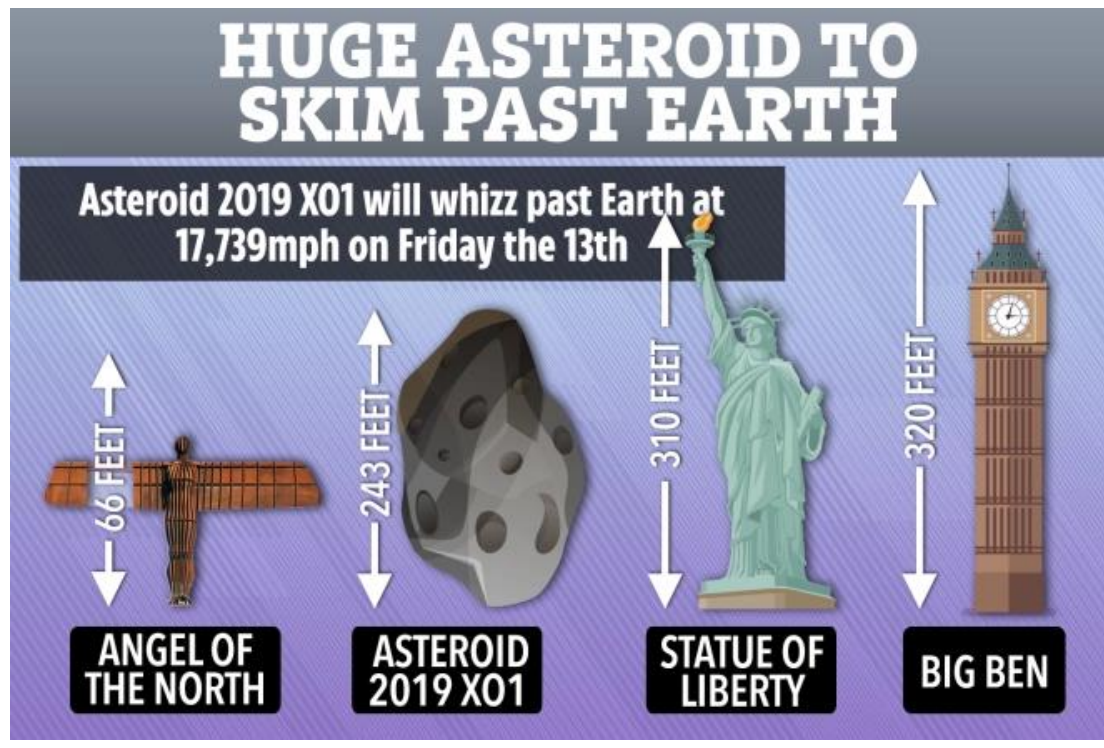

Image courtesy of "The Sun" December 13, 2019

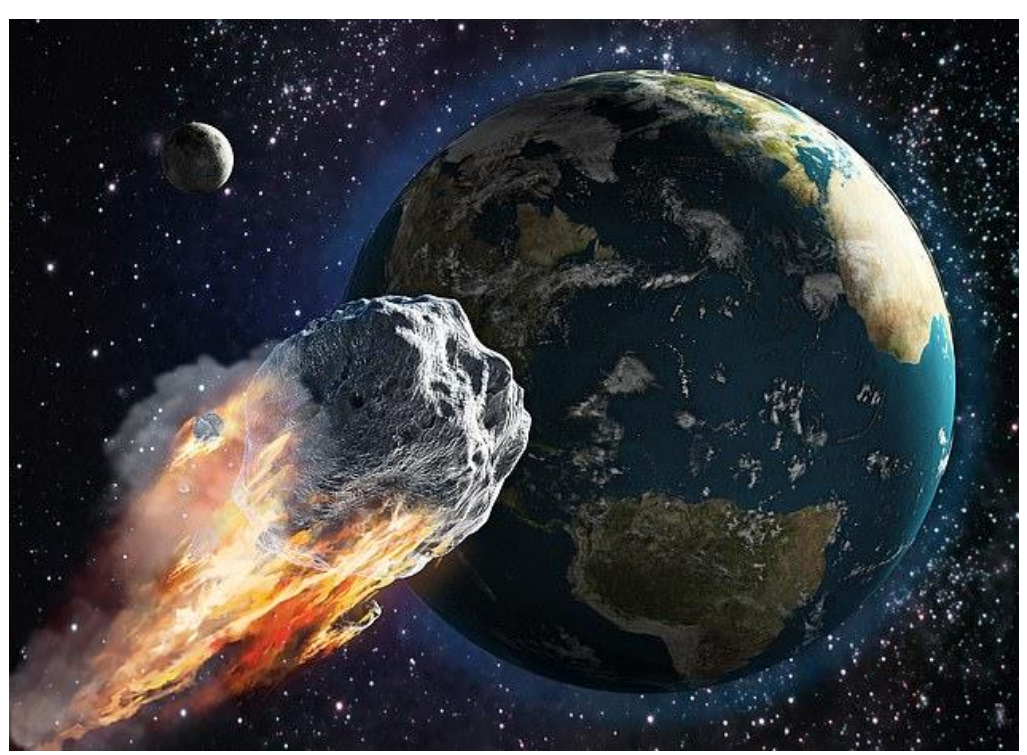




\section{Managing Accidents}

- Such emergency planning leads to a vicious cycle

- "Perception is reality" is not true in the real physical world

- "Reality is that which, when you stop believing in it, doesn't go away"

- Planning for only the big accident makes it seem more plausible, which in turn means that one had better plan for it

- Worst of all, the planners and operators soon come to believe it themselves

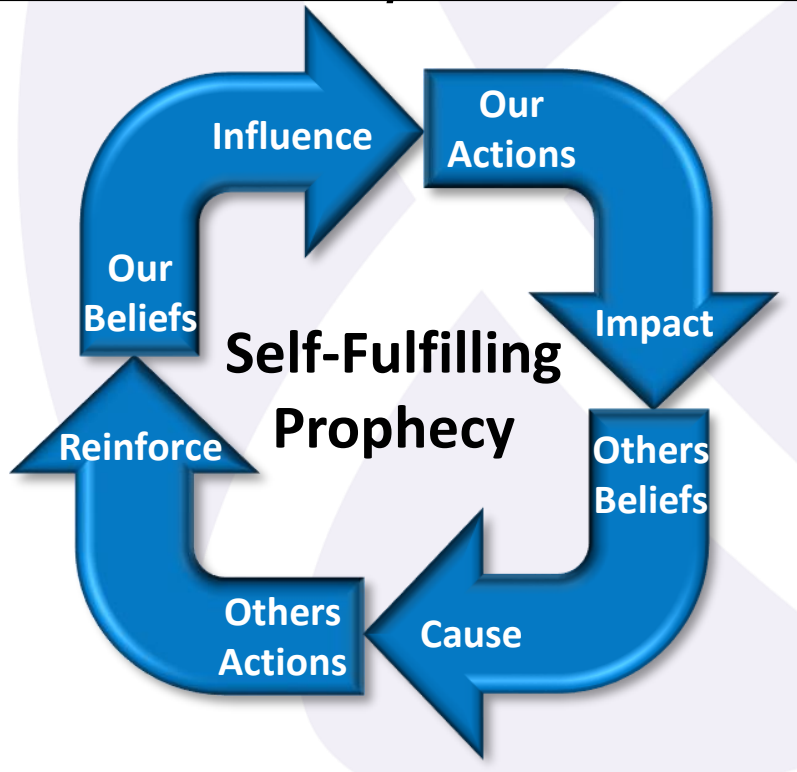




\section{Balance within Safety Culture}

Worker safety entails addressing a variety of hazards, some unique to nuclear process operations, but many more that are general.

Including, but not limited to:

Asbestos

Chemicals and toxic materials

Confined spaces

Cryogenics

Electrical

Ergonomics

Fire

Firearms

Hearing conservation

Ionizing radiation
Machine tools

Non-ionizing radiation

Nuclear criticality

Pressure

Radioactive contamination

Rigging and lifting

Stored energy

Thermal

Traffic and vehicles

Walking and working surfaces 


\section{Managing Risk}

- Avoid focusing solely on the worst-case scenario or the worst that can be imagined as they are typically also the least likely to occur

- Expends resources that limits the response options for more likely events

- Planning only for the worst leaves us less prepared for reality

- Example: LANL 2003 "Five Worker" event at the Plutonium Facility

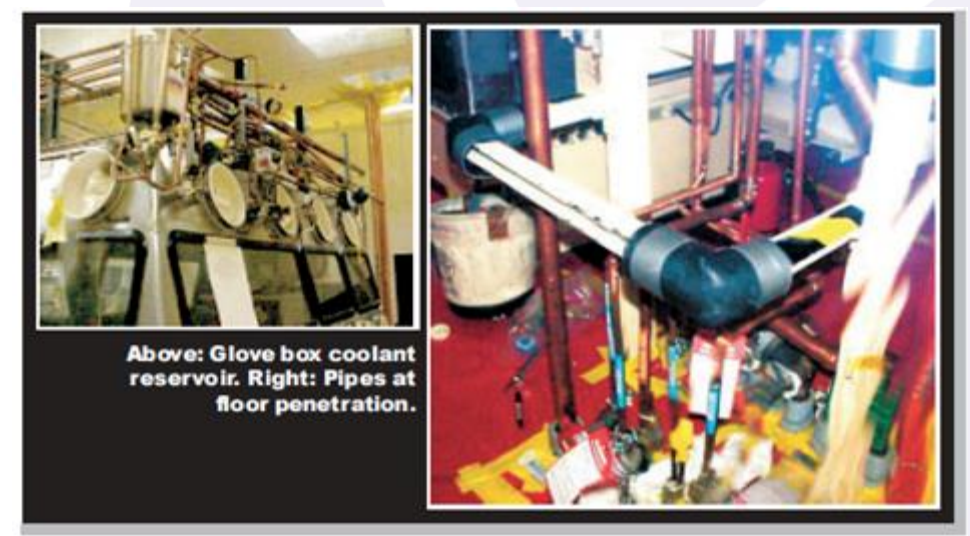

Five employees hospitalized after exposure to vapors 


\section{Safe Conduct of Research (SCoR) Principles}

1) Everyone is personally responsible for ensuring safe operations

2) Leaders value the safety legacy they create in their discipline

3) Staff raise safety concerns because trust permeates the organization

4) Cutting-edge science requires cutting-edge safety

5) A questioning attitude is cultivated

6) Learning never stops

7) Hazards are identified and evaluated for every task, every time

8) A healthy respect is maintained for what can go wrong 


\section{SCoR Principles Emphasis}

- "When everyone is in charge, no one is in charge" is the result of too many reviews and approvals before any work can be performed

- Excessive regulation and procedural layers do away with individual responsibility, thus decision making must be transferred from words on a page back to people on the job site

- In the current paradigm, no one has the ability to make decisions because excessive regulation, by attempting to foresee all possible situations and consequences, has supplanted the informed decision making process

- Replace layers of regulation and oversight with individual responsibility, hold the individuals accountable but liberate their judgment

- Return to workers being responsible for themselves 


\section{“Tickling the Dragon” Event}

- Qualified workers, but not just expert based, instead of extensive detailed operating procedures

- Example: LANL in 1946 Louis Slotin criticality accident, resident expert but not following the procedures he required of others
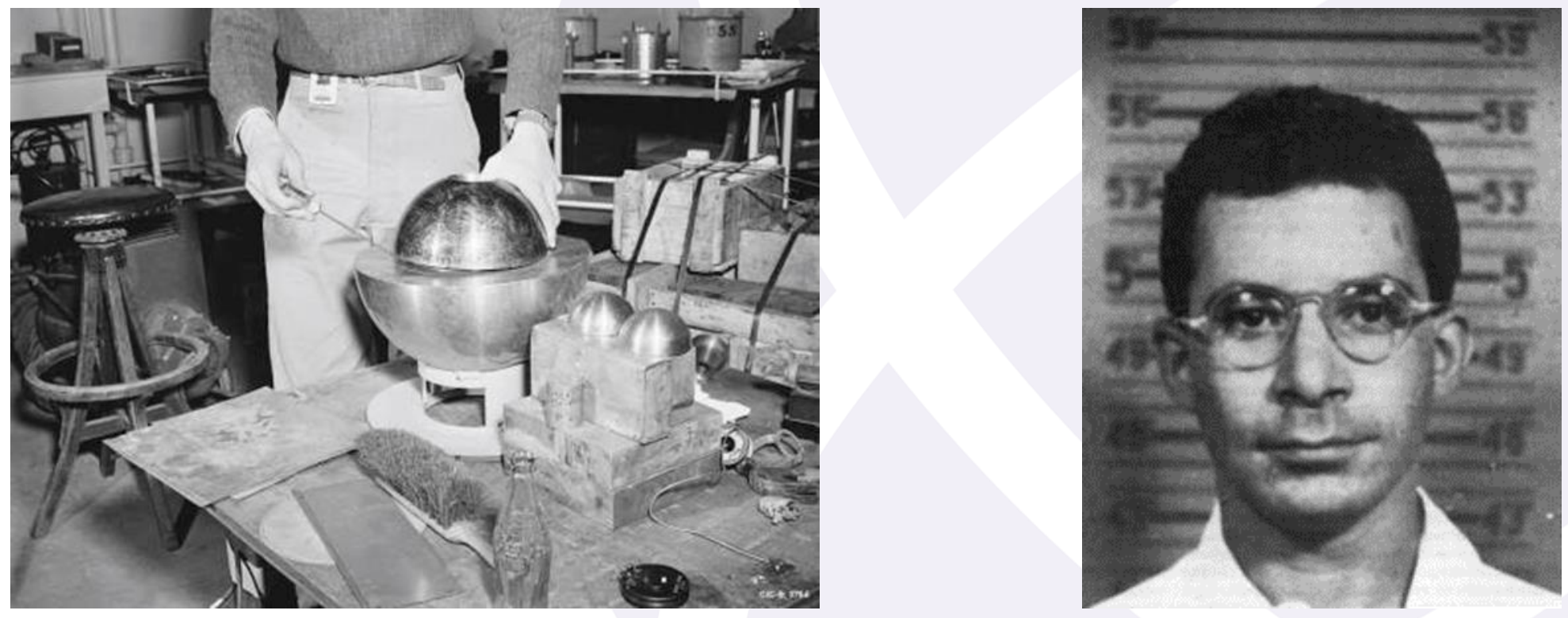


\section{SCoR Principles within Nuclear Safety Culture}

- Safety is a balancing act

- View safety as an evolutionary process rather than as a permanent condition because there is no stable optimum and there is always room for improvement

- Safety must be continuously re-accomplished or it will decline, but this may not be known until some bad event highlights it, so we must continuously learn from all events that deviate from expectation

- Safety requires ongoing vigilance

- Safety is relative; being safer than we used to be does not mean being as safe as we might be 


\section{ALDWP}

VALUES \& BEHAVIORS

\section{How we do work is as important as what we do.}

\section{VALUES}

\section{Commitment}

We are dedicated to successfully meeting the Laboratory nuclear security mission and associated deliverables demonstrating a shared commitment while exceeding the expectations

\section{Respect}

We will value differences and encourage every team

member to express themselves in order to work together toward common goals. We demonstrate high regard for customers, partners and colleagues as we work to meet to serve our Nation and Community.

\section{Integrity}

We will maintain high ethical standards. We are honest and fair in all aspects of our work. We fulfill our obligations as responsible employees and stewards of the taxpayer's dollar. We value our personal reputation and the reputation of the organization. We recognize that our integrity can impact national security

\section{Dealing With Problems}

We will anticipate, identify, acknowledge and take initiative

to understand the root cause and solve problems.

We acknowledge and learn from our mistakes without

assigning blame. We help others to solve problems and

will be responsible and accountable for our actions.

\section{Taking Care Of People}

We will recognize and value each person and continuously look

out for one another through respectful behavior and safe work practices to achieve the success of the individual, the Institution the mission

\section{Precision In Language}

We will take the time to formulate our thoughts before speaking and writing to accurately convey our message. We will maintain respectful language and approach in all interactions.

\section{B EHAVIOR S}

\section{Personal Responsibility}

We will have the courage to hold ourselves accountable
and behave in a professional manner demonstrating integrity, and behave in a professional manner demonstrating integnity
ethics and a high standard of values. We will demonstrate ethics and a high standard of values. We will demon
discipline while maintaining a questioning attitude.

\section{Value The Legacy}

We will respect the contributions of the past. In addition, we will actively seek the Knowledge and expertise of team members. We will share our collective knowledge to foster growth among

Raise Concerns

We will create a safe environment in which concerns can be raised without fear of reprisal. We will address problems, staff concern organizational obstacles and difficult situations respectfully and directly in a timely manner.

\section{Continuous Learning}

We will proactively develop, adapt and transform ourselves

through a mindset of continuous learning and share those

lessons with others. We will assess risks and emerging challenges to ensure they are addressed appropriately through a framework of informed perspectives.

\section{Continuous Improvement}

We will challenge the status quo, consider new ideas from anywhere and never become complacent with success. We w and continuously improve

Los Alamos 


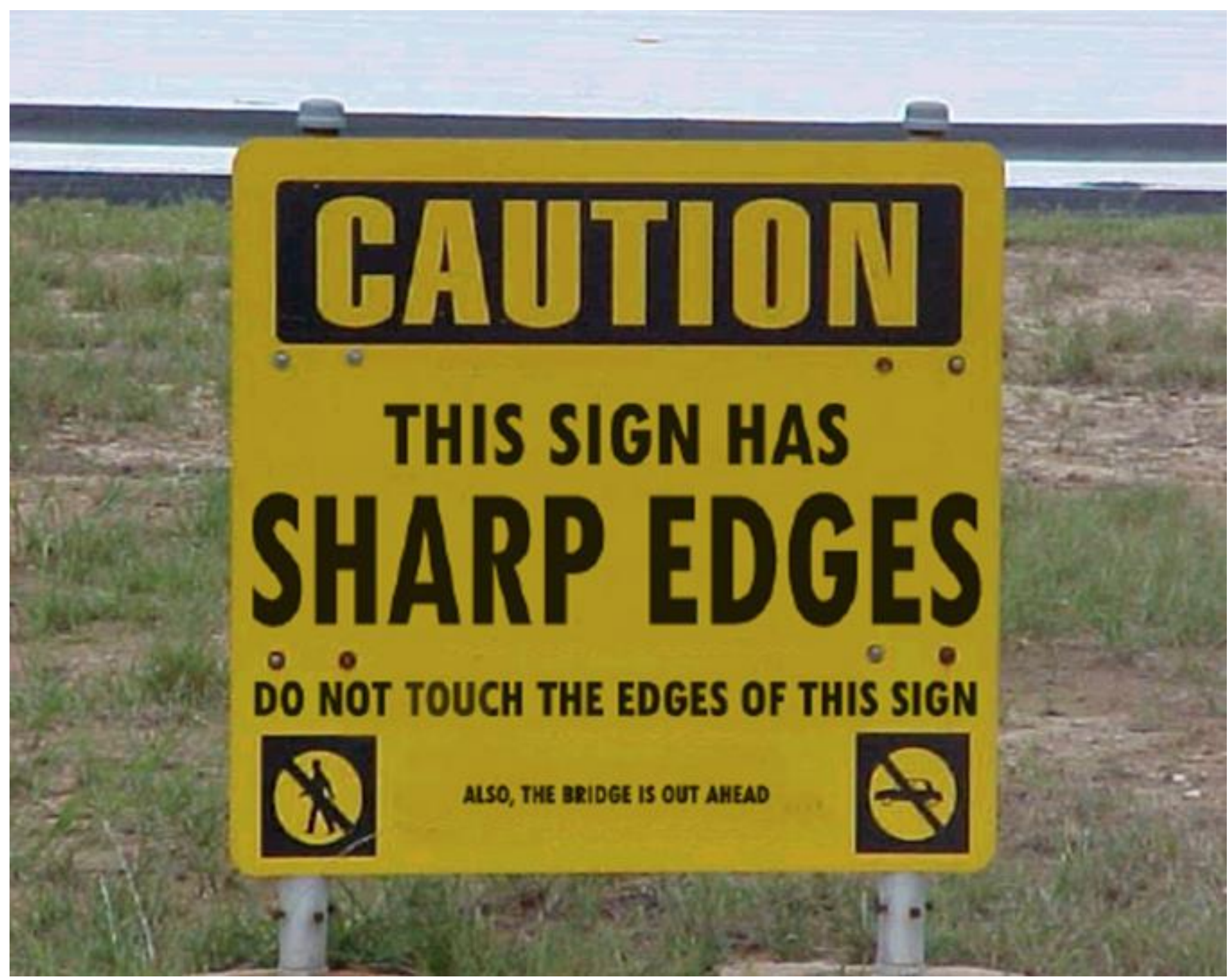

